\title{
Lidil
}

Revue de linguistique et de didactique des langues

35 | 2007

Figures de l'auteur en didactique

\section{Une séance d'histoire au Cycle 3}

La posture énonciative de l'enseignant dans la médiation documents/ élèves

Anne Leclaire-Halté

\section{(2) OpenEdition}

Journals

Édition électronique

URL : http://journals.openedition.org/lidil/2273

DOI : $10.4000 /$ lidil.2273

ISSN : 1960-6052

Éditeur

UGA Éditions/Université Grenoble Alpes

\section{Édition imprimée}

Date de publication : 1 juin 2007

Pagination : 139-157

ISBN : 978-2-84310-100-7

ISSN : $1146-6480$

\section{Référence électronique}

Anne Leclaire-Halté, « Une séance d'histoire au Cycle 3 », Lidil [En ligne], 35 | 2007, mis en ligne le 26 mars 2009, consulté le 01 mai 2019. URL : http://journals.openedition.org/lidil/2273; DOI : 10.4000/ lidil.2273

Ce document a été généré automatiquement le 1 mai 2019.

(C) Lidil 


\title{
Une séance d'histoire au Cycle 3
}

\author{
La posture énonciative de l'enseignant dans la médiation documents/ \\ élèves
}

\author{
Anne Leclaire-Halté
}

1 Dans ce numéro consacré à la construction de la figure de l'auteur, essentiellement dans des documents didactiques tels que les manuels scolaires, la spécificité de l'analyse qui suit, par rapport au contenu des autres articles, tient au fait qu'elle s'intéresse à l'oral plutôt qu'à l'écrit stricto sensu, à l'exploitation interactionnelle de documents plutôt qu'à celle d'un manuel, à l'école élémentaire plutôt qu'à l'enseignement secondaire ou supérieur, à l'histoire plutôt qu'au français.

2 Nous revenons en effet sur une séance d'histoire menée fin 2005 dans une classe de CM1$\mathrm{CM} 2$ de 29 élèves. Cette séance se réfère, à travers divers documents, à des personnages et évènements du début du Moyen-Age et se déroule en trois séquences successives.

3 - Des documents iconographiques affichés sous une frise chronologique présente sur les murs de la classe sont commentés (séquence A : 1 à 39). L'objectif de cette séquence est d'introduire, à partir de ces documents, la notion de représentation et de la mettre en rapport avec celle de source historique.

4 - Une comparaison entre deux documents iconographiques représentant le baptême de Clovis est proposée aux élèves (séquence $B$ : 39 à 104). Après la passation de la consigne par l'enseignant, les élèves examinent par binômes les documents puis une mise en commun est faite. L'objectif de cette séquence, pour l'enseignant, est d'amener les élèves à garder un esprit critique quand ils ont à traiter des documents, de quelque nature qu'ils soient.

5 - Ces deux séquences A et B forment un tout que l'on pourrait qualifier de méthodologique et elles précèdent une troisième séquence (séquence $\mathrm{C}$ ) où les élèves remplissent un questionnaire de comparaison entre trois documents (deux enluminures et un texte) sur la cérémonie de l'hommage au Moyen-Âge. Cette activité donnera lieu à une trace écrite dans un classeur, mais cette trace écrite ne sera pas élaborée le jourmême. L'objectif de C est essentiellement la construction de savoirs historiques à partir de documents. 
6 Je ne parlerai ici que des deux premières séquences (désormais $A$ et $B$ ), et je me centrerai plutôt sur B. Je n'ai ni transcrit ni traité la troisième, parce qu'elle était inachevée et parce qu'elle ne comportait pas de moment avec échange en groupe-classe. Or je m'intéresse, dans cette séance, à la posture énonciative de l'enseignant dans la médiation qu'il opère entre groupe-classe et documents, et aux problèmes qu'elle pose.

7 Dans l'analyse qui suit, je différencie, contrairement à G. Philippe (2000) et A. Rabatel (2004), la notion de centre interprétatif de celle de figure de l'auteur ou d'archiénonciateur. G. Philippe distingue, dans les études romanesques, les théories qui privilégient le centre énonciatif des textes ${ }^{1}$ de celles qui mettent en avant le «centre interprétatif, conçu comme une figure de l'auteur, et tentent de voir comment ce centre interprétatif interagit avec le texte et lui confère un sens global » (p. 38). Il distingue ainsi centre interprétatif et sens global. A. Rabatel, pour qui la notion de centre interprétatif peut être rapprochée de ce qu'il nomme, en s'inspirant de D. Maingueneau (1990), la posture archi-énonciative, écrit de cette dernière qu'elle «met en jeu l'ensemble des mises en rapport qui forment le substrat méthodique et notionnel des activités cognitives» (p.63). Pour ma part, je nomme centre interprétatif le substrat méthodologique ou notionnel construit au cours de la séance, c'est-à-dire le sens global de cette dernière, pour reprendre les termes de G. Philippe. Quant à la figure de l'auteur ou l'instance archi-énonciative, elle serait celle qui hiérarchise et les informations livrées par les documents et les informations provenant des interactions à propos de ces documents, pour reformuler les points de vue en objet de savoir. Ce devrait être la posture que l'enseignant assume (ou construit chez les élèves) dans une séance d'histoire où des documents sont à traiter. Le rôle de l'enseignant est en effet d'orchestrer, ou de faire orchestrer par les élèves (selon le type de pédagogie adoptée), les différents points de vue qui se font entendre lors de la séance, ceux des élèves, des auteurs des documents, celui de l'enseignant, de façon à orienter la séance vers la construction d'un substrat précis, qui constitue le centre interprétatif de la séance. Quel est celui-ci dans cet exemple? L'enseignant occupe-t-il une posture archi-énonciative? En vise-t-il la construction chez les élèves?

8 Je développerai trois points :

- le centre interprétatif de A et B ;

- la manière dont le centre interprétatif contraint le choix des documents proposés aux élèves ;

- l'exploitation interactionnelle des documents.

\section{Le centre interprétatif des deux séquences}

9 À la fin de la séquence B, l'enseignant explicite le fil conducteur des deux séquences, qui est un raisonnement et qui constitue en fait le substrat de A et B :

Extrait de transcription 1

11 102- $\mathrm{M}^{2}$ on a vu deux représentations différentes d'un même évènement / d'une même scène qui pouvait avoir des points communs mais aussi des différences / d'accord / donc qu'est-ce que ça veut dire quand on va consulter des documents qui se trouvent dans un manuel ou des documents historiques? Il faut faire attention à quoi ? (nous soulignons)Baptiste

103-B (inaudible) exact... 

hommes des personnages qui représentent l'église / des évêques / sur l'autre document il $\mathrm{y}$ a d'autres personnages à gauche de Clovis en plus / donc comment savoir nous quelle est la vérité ? est-ce qu'il y avait uniquement l'évêque réuni et d'autres personnages de l'église ou est-ce qu'il y avait en plus des seigneurs ou des gens de la famille de Clovis? Maintenant comment savoir est-ce que Clovis portait une barbe / c'est peut-être qu'un détail / mais portait-il une barbe / ne portait-il pas de barbe ? quelle était la forme de sa couronne? / quelle était la forme du baptisphère etc. ? / donc il y a des ressemblances mais il y a quand même des différences / il faut faire attention / ce sont des représentations / je répète / ce sont des représentations qui ont été faites après cet évènement / après le baptême de Clovis / donc les personnes qui ont réalisé ces miniatures ou ces enluminures ont commis des inexactitudes / bon alors pourquoi je vous ai fait faire ça?

On s'aperçoit, dans cette phase conclusive, se présentant comme du discours magistral, qu'il ne s'agit pas pour l'enseignant que les élèves se construisent des savoirs sur une période donnée. Ses visées relèvent plutôt de la méthodologie. Quelle est l'orientation argumentative générale du propos? Il y a une vérité mais elle est inaccessible dans les documents. Si deux documents représentant le même évènement offrent des différences, c'est qu'ils comportent des inexactitudes, et s'ils comportent des inexactitudes, c'est qu'ils ne sont pas conformes à la vérité. Et s'ils ne sont pas conformes à la vérité, c'est qu'il s'agit de représentations, postérieures aux faits.

tours de parole 102 et 104, certains propos de l'enseignant sont à plusieurs reprises marqués du sceau de l'effacement énonciatif ${ }^{3}$. En 102, les deux énoncés interrogatifs, avec leurs indéfinis génériques, leurs formules impersonnelles ("ça veut dire », « il faut »), la présence de " on », marquent un décrochage énonciatif par rapport aux propos précédents et introduisent la question fondamentale et sa réponse. Même si les interrogations et les énoncés généralisants qui suivent sont mêlés à des énoncés convoquant à titre d'exemples des éléments présents dans la situation d'énonciation, ils sont signes d'une "objectivation » conclusive de l'énonciateur par rapport à tout ce qui s'est dit en A et B. Il s'agira par la suite de voir dans quelle mesure les élèves et les documents participent à cette conclusion.

Un entretien avec l'enseignant après visionnement de la séance met en évidence que son objectif est de former l'esprit critique de ses élèves : ils doivent être en mesure de prendre de la distance par rapport aux documents qu'ils lisent. L'enseignant dit avoir conçu A et B plus dans l'optique de l'éducation à la citoyenneté que de l'histoire proprement dite. En se donnant cet objectif, il est aussi en conformité, même s'il ne le formule pas, avec les documents d'application des programmes préconisant de commencer à former l'esprit critique des élèves ${ }^{4}$. On constatera en tout cas que le centre interprétatif, c'est-à-dire le substrat de la séance, est en fait un raisonnement menant à une mise en garde ( $«$ il faut faire attention »), et non des savoirs historiques issus de la confrontation et de la hiérarchisation de points de vue repérables dans les documents et dans les propos des élèves.

La construction de la séance par rapport à ce centre interprétatif se lit en filigrane dans l'agencement de la séance, dans l'enchainement de A et B. J'ai précisé plus haut que A est une séquence visant à établir ce qu'est une représentation (bien que cela ne soit jamais vraiment explicité, l'enseignant fait apparemment une distinction entre représentation et source historique qui correspond à celle existant entre source primaire et source 
secondaire). La notion de représentation est effectivement construite en A pour arriver ensuite à montrer en $\mathrm{B}$ le peu de fiabilité de ces dernières.

En A, les élèves reviennent sur des documents affichés sous certaines dates dans une frise chronologique murale. Un décalage est constaté par certains entre telle date de la frise et telle date d'exécution du document affiché :

\section{Extrait de transcription 2}

9-M en 496 s'est passé le baptême de Clovis et vous m'avez posé / y a Manon qui m’a posé une question ce matin / qui m'a dit ben oui mais là c'est marqué xve siècle en dessous du document / donc Manon tu penses qu'il fallait le mettre plutôt où ? Ta première idée c'était de le mettre où?

10-Ma au XV siècle

11-M oui on a réfléchi et qu'est-ce qu'on a dit ensuite ? Baptiste ?

12-B ben que y a les gens ils ont représenté après / mais ils étaient pas là / par exemple quand on a fait l'enluminure du baptême de Clovis / il était pas au baptême / alors celui qui a fait l'enluminure et puis euh il a un petit peu imaginé (inaudible) / y a pas que l'imagination y a eu aussi des fouilles archéologiques

13-M donc tu as dit que c'était une représentation d'accord / c'est quelqu'un qui a représenté la scène du baptême de Clovis / donc c'est bien je l'avais pas utilisé et toi tu l'as bien trouvé / donc y a certains documents qui sont des représentations / notamment cette enluminure là qui a été réalisée après le baptême de Clovis plusieurs siècles après d'accord / qui n'a pas été faite le jour du baptême de Clovis / Elise

En 12, Baptiste fait un rappel de ce qui a été dit avant, à un autre moment en classe : des évènements sont représentés postérieurement à l'évènement et donc de manière non objective. En 13, l'enseignant procède à une généralisation par nominalisation (« donc tu as dit que c'était une représentation ») répétée avec effacement énonciatif ( y a certains documents qui sont des représentations »). Il se positionne ici en tant que surénonciateur (Rabatel, 2004) soucieux d'une mise en ordre cognitive au niveau de A. 12 et 13 sont à mettre en relation avec 104, où le lien et la progression entre $\mathrm{A}$ et $\mathrm{B}$ apparaissent : « ce sont des représentations qui ont été faites après cet évènement / après le baptême de Clovis / donc les personnes qui ont réalisé ces miniatures ou ces enluminures ont commis des inexactitudes $"$ : représentation et inexactitudes sont plus nettement associées par l'enseignant.

Mais cette posture de sur-énonciateur n'est pas tenue jusqu'à la fin de A. L'enseignant demande aux élèves de se prononcer sur une statue en bronze de Charlemagne du Ixe siècle mise sous la date 800 : source historique ou représentation? La séquence se clôt sans qu'une réponse claire soit donnée, le seul critère de postériorité associé par l'enseignant à la notion de représentation lui posant problème :

Extrait de transcription 3

22-J Charlemagne c'est une statue de bronze

\section{3-M Oui}

\section{4-J qui a été faite}

25-M donc c'est une représentation ou c'est une source historique?

26-J c'est une source historique

27-M elle date du IX siècle d'accord donc Charlemagne dans quel siècle a-t-il vécu? 

est pas daté précisément / mais il n'a pas été réalisé vraiment / il a été réalisé dans le siècle pendant lequel a vécu Charlemagne / alors ce que j'ai fait moi / j'ai regardé dans différents manuels et puis j'ai retrouvé plusieurs enluminures / donc deux enluminures/ pas plusieurs / deux tout simplement / qui représentaient le même évènement/enfin la même scène notamment le baptême de Clovis/donc ce que je vais vous demander de faire/c'est de les/je vais distribuer les manuels d'histoire et dans lequel se trouve une enluminure qui représente le baptême de Clovis / et j'en ai photocopié une autre / qui représente exactement la même scène et je vais vous demander de (interruption : une élève vient demander des manuels) donc je vais vous demander de comparer ces deux enluminures qui représentent la même scène d'accord/et d'essayer de voir donc les points communs qu'il peut y avoir ou les différences / donc sur deux représentations du même évènement de la même scène / donc je vous distribue un livre / ouvrez-le à la page 34 et en même temps je vais vous distribuer une photocopie que j'ai trouvée dans un autre manuel de la même scène du baptême de Clovis/on va essayer de voir les différences et les ressemblances/les points communs et les différences

Là où on attendrait une prise de distance par rapport à l'ensemble de la séquence A pour la recentrer, pour faire une synthèse intermédiaire, on constate que cette posture n'est pas tenue. Pour comprendre l'objet du travail et structurer l'avancée de la séance, les élèves auraient eu besoin de cette mise au point.

Il est à remarquer aussi que si l'observateur peut construire à rebours, en quelque sorte, le lien entre $A$ et $B$, en ayant connaissance de la clôture de $B$, il semble bien difficile pour les élèves d'identifier l'enchainement entre $A$ et $B$ à partir de la transition qu'opère l'enseignant dans le tour de parole 39 : il enchaine une non-réponse à sa propre question en 25 à une consigne très répétitive de comparaison de deux enluminures entre elles. Les élèves vont-ils faire un lien entre A et B ? C'est peu probable pour un certain nombre d'entre eux. Si l'enchainement apparait nettement à l'observateur extérieur en possession de la totalité des deux séquences, il apparait insuffisamment relayé par une sur-énonciation qui devrait être plus marquée en fin de A et dans la transition entre A et B.

Il a été souligné que le centre interprétatif était d'ordre méthodologique plus qu'historique. Il s'agit maintenant d'étudier la gestion des documents par rapport à ce centre interprétatif : comment ont-ils été choisis ? Comment l'enseignant gère-t-il leur exploitation dans l'interaction? Adopte-t-il une posture archi-énonciative ou permet-il aux élèves de se la donner?

\section{Le choix et la situation des documents : I'influence du centre interprétatif}

Beaucoup d'enseignants du premier degré n'utilisent pas de manuels, mais s'appuient sur des documents d'origine diverse qu'ils vont chercher ici et là. C'est le cas dans cette 
séance. Faute de place, je ne parlerai que des documents traités dans B (voir annexe). Pourquoi ces documents? Pourquoi des documents aussi proches?

Les deux enluminures supports de l'activité comparative proposée aux enfants représentent un évènement idéologiquement chargé et sujet à polémique : le baptême de Clovis $^{5}$. Les historiens contemporains montrent bien comment le glissement du baptême au sacre s'opère à travers les siècles, dans l'évocation de cet évènement religieux(voir Le Goff 1986$)^{6}$. Les manuels les plus récents essaient d'éviter l'amalgame entre le baptême de Clovis, évènement religieux, et la royauté en donnant aux élèves, pour évoquer cette période, des documents tels que des vestiges de l'art franc ou alors des documents évoquant le baptême mais sans association avec le couronnement.

L'enseignant, lui, donne aux élèves deux documents très proches, des enluminures tirées des Grandes Chroniques de France, écrites du XIIIe au xvie siècles, et destinées à établir en français la mémoire dynastique : cet ouvrage constitue en effet la matrice d'une histoire monarchique, française et chrétienne. La première enluminure apparait dans le manuel scolaire disponible à l'école (Pour connaitre la France, 1985, Histoire CM, Hachette classiques), elle est simplement légendée «le baptême de Clovis ». Nulle indication ni de date, ni de source. En fait, elle date du xive siècle et est tirée des Chroniques. On y voit Clovis nu et plongé dans les fonts baptismaux. À ses côtés, se trouvent un évêque et Saint Rémi. Rémi, reconnaissable à sa mitre et à sa crosse, verse l'eau du baptême sur la tête du roi. Au-dessus de la tête de Clovis, une colombe, envoyée par Dieu, et représentant l'esprit saint, apporte la sainte ampoule qui contient l'huile sacrée (le saint chrême, mélange d'huile d'olive et de résines aromatiques) pour oindre le roi. Clovis porte une couronne qui est le symbole de son pouvoir. Cette enluminure est un moyen de propagande monarchique qui montre les rois francs en contact direct avec Dieu. Le baptême de Clovis et le sacre des rois de France sont assimilés : l'Église a conçu, en souvenir du baptême de Clovis, la cérémonie du sacre, durant laquelle l'évêque déposait de l'huile sainte ${ }^{7}$ sur le front des rois de France, pour montrer que leur pouvoir venait de Dieu.

Le deuxième document est pris dans un autre manuel par l'enseignant comme il le mentionne en 39. Il est légendé de façon plus précise (Les Grandes Chroniques de France, $\mathrm{XIV}^{\mathrm{e}}$ - $\mathrm{xV}^{\mathrm{e}}$ siècles), et, s'il présente quelques différences de détail avec le premier (nous y revenons dans la partie suivante), il est porteur des mêmes symboles et de la même idéologie.

Ces documents appellent plusieurs remarques :

- ils présentent plusieurs éléments symboliques (comme la couronne, la colombe, la sainte ampoule par exemple) à commenter si l'on veut qu'ils soient compris des élèves ;

- ils nécessitent un recadrage quant à leur source pour en saisir la portée idéologique (tous les deux amalgament royauté et catholicisme);

- ils seraient à confronter avec d'autres documents représentant la même scène sans être porteurs de la même idéologie.

Or on constatera dans la troisième partie que l'enseignant n'exploite pas ces documents dans leur dimension historique et idéologique et qu'il ne cherche pas à aider les élèves à accéder à leur centre interprétatif.

Pourquoi choisit-il alors ces documents?

- d'abord parce qu'ils font référence à un évènement du Moyen-Age qui est au programme ;

- ensuite parce qu'ils permettent d'enrichir le vocabulaire des enfants; 
- enfin et surtout parce que le centre interprétatif de la séance étant que deux représentations du même évènement ne sont pas identiques et sont donc sujettes à caution, il a choisi simplement deux documents très proches, à partir desquels on peut jouer à ce qui s'apparente à un jeu des ressemblances et des différences. Mais ce jeu, qui aurait pu par ailleurs se faire à partir de deux cartes postales représentant de façon différente la même ville, n'est possible qu'au prix d'un double effacement idéologique. Les documents ne sont pas replacés dans une perspective diachronique qui permettrait la confrontation des points de vue selon les époques. Et, lors de leur exploitation en classe, dans l'activité comparative, toute tentative interprétative des élèves est en quelque sorte cassée, comme les lignes qui suivent le montrent.

\section{L'enseignant : quelle interface entre les documents et les élèves?}

L'enseignant assure l'interface entre les documents et les élèves de manière à mener ceux-ci au centre interprétatif mis en avant dans la première partie : il faut se méfier des représentations, n'étant pas contemporaines des faits, elles présentent des inexactitudes. Pour atteindre cet objectif, il n'a besoin que de leur faire relever qu'il y a des différences et des ressemblances entre les deux enluminures et pour cela il affiche en exemple et il demande aux élèves une position d'objectivité, de « scientificité ». Et pour lui, objectivité est synonyme d'extériorité, de description en surface, de refus d'interpréter. Les documents ne sont pas considérés comme porteurs de points de vue à traiter et à confronter, pour se positionner en tant qu'interprète, mais comme des objets à comparer en surface. Plusieurs tours de parole et échanges montrent les différentes manières dont l'enseignant cherche à imposer son centre interprétatif, malgré les résistances de certains élèves.

\section{Le retour sur l'identification des documents}

Après la présentation des documents et la passation de la consigne, les élèves travaillent par deux puis la mise en commun commence.

\section{Extrait de transcription 4}

47-M quand quelqu'un prend la parole on dit pas / c'est pas ça / c'est pas ça / on écoute tout le monde / et puis si on veut lui répondre on lève le doigt et puis on lui répond / alors Gilles

48-G là y a le roi qu'est dedans ben

49-M dis-nous déjà/essayez de nommer les documents/vous avez le document un / le document du manuel / avant de l'interroger qu'est-ce nous dit le manuel sur ce document? est-ce qu'on nous dit quand il est daté et ce que c'est exactement? est-ce qu'on peut deviner ce que c'est? je vous interrogerai après / je voudrais au préalable vous demander ça / Baptiste?

50-B ben on voit que c'est un baptême

51-M donc c'est marqué le baptême de Clovis

52-B même si c'est pas marqué

53-M sur le document un du manuel 

un document sur celui que je vous ai distribué on voit un peu la date de réalisation / on sait que c'est une enluminure / sur le document du dessus on nous donne seulement le titre / maintenant si on compare moi je suppose que c'est une miniature ou une enluminure aussi / d'après la forme des personnages d'après etc. / alors si on compare ces deux documents quels sont les points communs et quelles sont les différences? Gilles vas-y / vous écoutez Gilles

L'enseignant donne la parole à Gilles en 47, comme il le lui a annoncé en 46. Mais à peine Gilles commence-t-il à décrire l'image qu'il est interrompu en 49 : l'enseignant demande aux élèves de nommer les documents, c'est-à-dire en fait de lire la légende, en une séquence latérale de 47 au début de 70 . Ensuite cette dernière est close par une relance vis à vis de Gilles à la fin de 70, ce qui indique que l'enseignant exerce une certaine maitrise dans la progression de sa séquence. Dans cette vaste séquence latérale, l'imprécision 
d'une légende est signalée, mais non remise en cause. L'enseignant joue même le jeu de l'ignorance en 66.

Alors que son rôle devrait être de donner l'information manquante ou d'inciter les élèves à la chercher, il se donne comme prenant le document tel qu'il est, sans désir d'ajouter de l'information, en une sorte de retrait objectif par rapport aux données. Et c'est l'attitude générale qu'il favorise par rapport aux documents, comme on le vérifiera par la suite.

\section{La préoccupation lexicale}

77 Le souci métalinguistique d'enrichir le lexique historique des élèves est très présent. L'enseignant veut être en conformité avec un des objectifs de la séance d'histoire selon les nouveaux programmes; mais on peut aussi trouver dans cette préoccupation de donner le mot juste pour référer à tel objet une préoccupation de scientificité. Par exemple, en 66 (voir plus haut), à partir du mot «miniature », l'enseignant développe une parenthèse définitoire sur la miniature et l'enluminure.

Parfois encore apparaissent des énoncés de dénomination, l'enseignant posant des questions dont il donne la réponse (en $82 / 84$ ou en $84 / 86$ ).

\section{Extrait de transcription 5}

82-M c'est des évêques d'accord / comment ça s'appelle les chapeaux? la coiffe? / Antoine retourne-toi

83-E pour un point commun

84-M réponds à la question ça s'appelle des mitres d'accord? donc qui sont ces personnages-là grâce à leur habillement ?

85-E ils baptisaient les gens

86-M ce sont des gens de l'église / des représentants de l'église / notamment des évêques d'accord? est-ce qu'on les trouve sur les deux documents?

Dans les deux cas, il y a une tendance à l'effacement énonciatif : l'activité définitoire de l'enseignant relève de la sur-énonciation, l'enseignant soulignant ponctuellement ce qui mérite d'être retenu de la leçon.

\section{L'occultation du symbolique et de l'idéologique}

86 À aucun moment ne sont commentés ou même évoqués les éléments qui pourraient poser problème aux enfants ou qui pourraient être l'objet de questions ou d'interprétations diverses: le fait que ce soit un adulte qu'on baptise, le rapport entre le baptême et la couronne, la colombe, la sainte ampoule par exemple. Or, par exemple, quand les élèves travaillent par deux, certains élèves pointent du doigt ce dernier élément et en parlent. De même, il n'est pas question des Grandes Chroniques de France ni du projet dont elles sont porteuses.

\section{Le refus de la parole subjective}

87 La sur-énonciation qui assure la progression vers la conclusion de la séquence est synonyme de coup de force de la part de l'enseignant. En effet, pour construire son propre centre interprétatif qui est d'ordre méthodologique et qui implique la négation de 
toute substance aux documents, le maitre rejette toute tentative d'interprétation qui impliquerait de la subjectivité de la part des élèves. document. de Clovis?» écoutez Gilles baptisé

\section{L'enseignant désire éviter une lecture plutôt esthétisante des documents}

En 43, l'enseignant met en garde contre des remarques à caractère esthétique, il les anticipe comme un bruit qui perturberait son cours. Certes, cette interdiction peut se comprendre et être interprétée comme un contrat didactique passé avec les élèves, pour délimiter l'objet d'étude. Mais aussi, à l'approbation en 45 qui suit la remarque faite en 44 par un élève, on voit que cette interdiction relève aussi du désir de rester dans l'objectif, de s'en tenir aux objets, dénués de toute valeur esthétique ou symbolique :

\section{Extrait de transcription 6}

43-M comme différence ne me dites pas / une enluminure celle du dessus tire plus vers les bruns / celle du dessous tire plus vers les bleus d'accord / essayez de

44-E c'est les objets

45-M voilà c'est bien

Il refuse d'entendre un élève qui convoque son expérience du monde pour interpréter un

Dans l'échange 49-55 (extrait de transcription 4), Baptiste reconnait une cérémonie du baptême sur l'enluminure, que cela soit écrit ou non. L'enseignant n'écoute pas sa lecture du document et ne veut qu'on rappelle que ce qui est marqué, ce qui est donc "objectif » pour lui. L'élève conteste à deux reprises sa réponse 51, en 52 et 54 . Mais l'enseignant ne l'entend pas et continue par un oui en 55 qui n'entérine pas réellement la remarque de Baptiste, puisqu'il maintient que l'information doit être tirée de la légende et non de ce qu'on voit : « oui / mais quelles informations écrites on nous donne mis à part le baptême

\section{L'enseignant refuse d'entendre l'attente historique des élèves par rapport à la séance.}

L'ensemble de la séance est présenté comme consacré à l'histoire, et la classe travaille sur des documents historiques. Certains élèves, par leur intervention, indiquent leurs attentes disciplinaires. Ils aimeraient qu'on leur apporte des connaissances historiques, comme on le voit dans l'échange suivant :

\section{Extrait de transcription 7}

70-M alors c'est une enluminure qui est datée du xive ou du xve siècle d'accord ? / alors un document sur celui que je vous ai distribué on voit un peu la date de réalisation / on sait que c'est une enluminure / sur le document du dessus on nous donne seulement le titre / maintenant si on compare moi je suppose que c'est une miniature ou une enluminure aussi / d'après la forme des personnages d'après, etc. / alors si on compare ces deux documents quels sont les points communs et quelles sont les différences? Gilles vas-y / vous

71-G sur le document de la feuille on voit où Clovis est dedans / on voit qu'il y a un bac / et sur le document que le maitre nous a distribué y en a aussi un / c'est pas les mêmes / mais y en a où il est

72-M qu'est-ce que ça t'apprend?

73-G ben que dans le temps ils se faisaient baptiser dans / dans ça 
74-M tu as appelé ça un bac / ça s'appelle un baptisphère d'accord / c'est un bassin dans lequel se trouvaient les gens qu'on allait baptiser d'accord / est-ce que la forme du bassin ou du baptisphère est identique / y a une différence Manon

En 70, l'enseignant encourage les élèves à comparer les deux documents distribués. Gilles remarque que sur les deux il y a un bassin, et pour lui, de la comparaison, on tire de l'information sur la façon dont se déroulaient les baptêmes autrefois, comme il le dit en 73. Mais ce n'est pas la réponse attendue puisque l'enseignant ne revient pas sur la généralisation de Gilles. En 74, après avoir dénommé et défini l'objet évoqué par l'élève, il relance la question sur les ressemblances et les différences parce qu'il s'agit de sa préoccupation majeure, plus que l'apport de connaissances historiques.

On ne peut donc dire que l'enseignant occupe une posture d'archi-énonciateur. Il ne prend pas en compte le point de vue des documents et dans de nombreux cas les élèves ne sont pas vraiment entendus. On ne peut parler ni de co-construction des savoirs ni de modèle de posture archi-énonciative donné aux élèves. Le centre interprétatif tel qu'il a été repéré dans la première partie n'est en fait qu'une construction auto-générée par l'enseignant et en décalage avec la nature des documents, ce qui explique la résistance de certains élèves.

En étant au plus près des pratiques réelles, ce genre d'analyse microdidactique permet d'approcher les composantes de l'expertise enseignante ordinaire et de mieux comprendre les réussites et les échecs conceptuels des élèves. Que tirer de l'étude de cette séance ordinaire de classe, nullement expérimentale ou exemplaire?

107 Dans les deux séquences observées, le choix de l'enseignant de séparer le méthodologique du disciplinaire se révèle malheureux, car il pervertit et la sélection et le traitement des documents. La distorsion entre le centre interprétatif des deux séquences et ce qui pouvait être tiré des documents conduit à une dilution dans l'anecdotique que l'on trouve en 104 : Clovis portait-il une barbe ou pas? Quelle était la forme du bassin? Qui était là le jour du baptême? Aucune réponse n'est de surcroit donnée à ces questions, dont on peut se demander ce que les élèves peuvent faire. L'enseignant ne traite correctement ni la dimension historique, ni la dimension méthodologique qui devrait impliquer le repérage de la source énonciative des documents pour les situer idéologiquement.

Alors même que l'enseignant se fixe comme objectif de former les élèves à la lecture distanciée des documents, en aucun cas il ne leur apprend à se construire une posture d'archi-énonciateur hiérarchisant les informations livrées par les documents de façon à reformuler les points de vue en objets de savoir.

\section{BIBLIOGRAPHIE}

\section{Références bibliographiques}

Guibert, N. (octobre 1996) : Clovis, ou quinze minutes de la vie d'un élève, Le Monde de l'éducation, 23-28. 
Le Goff, J. (1986) : Reims, ville du sacre, Les lieux de mémoire, sous la direction de Pierre Nora, t. II La nation, Gallimard, Paris.

Maingueneau, D. (1990) : Pragmatique pour le texte littéraire, Bordas, Paris.

Ministère de la jeunesse, de l'éducation nationale et de la recherche (2002) : Histoire et géographie cycle des approfondissements, coll. «École », Documents d'application des programmes.

Philippe, G. (2000) : Centre énonciatif et centre interprétatif - l'analyse linguistique et l'interprétation du roman, Études romanesques, 6, 37-52.

Rabatel, A. (2004) : Déséquilibres interactionnels et cognitifs, postures énonciatives et coconstruction des savoirs : co-énonciateurs, sur-énonciateurs et archi-énonciateurs, Rabatel, A. (éd) Interactions orales en contexte didactique. Mieux (se) comprendre pour mieux (se) parler et pour mieux (s')apprendre, Presses Universitaires de Lyon, Lyon, 26-69.

Vion, R. (2001) : “ Effacement énonciatif » et stratégies discursives, De la syntaxe à la narratologie énonciative, De Mattia, Monique et Joly, André (éds), Ophrys, Gap, Paris, 331-354.

\section{ANNEXES}

\section{Documents proposés aux élèves}

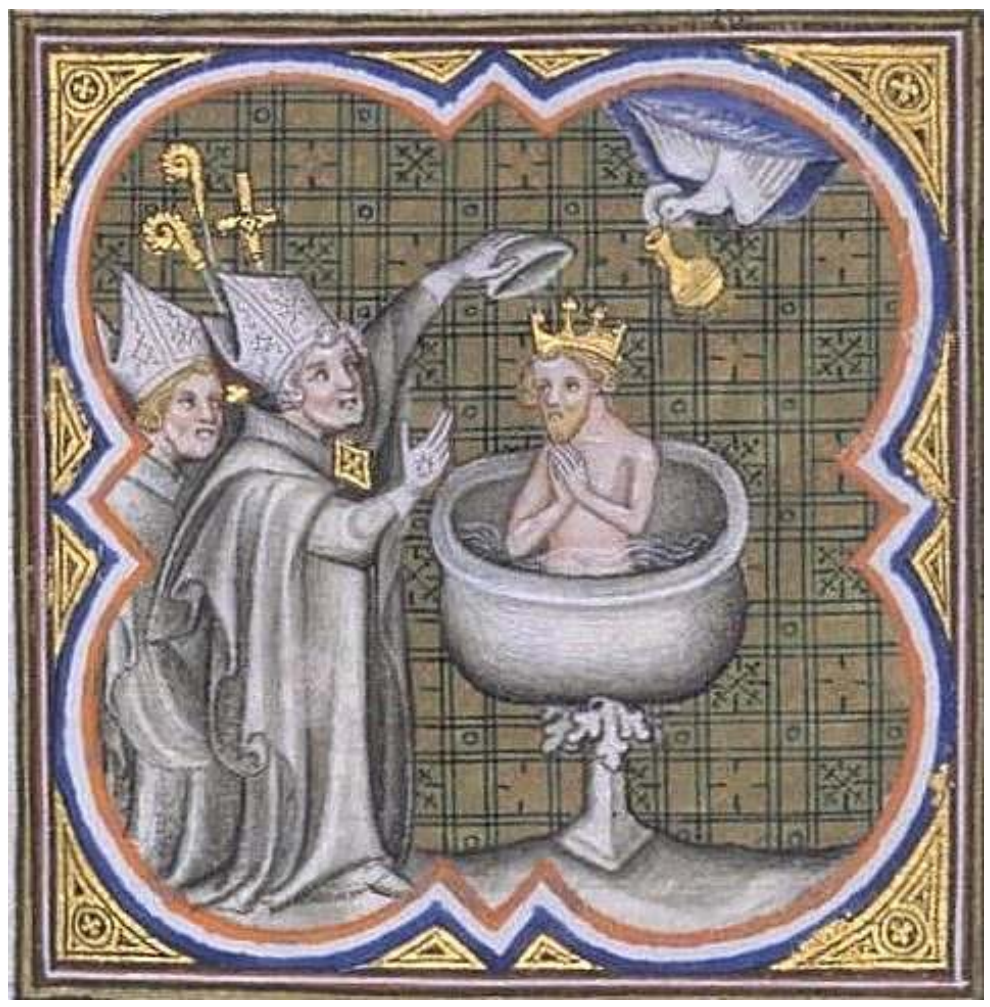

Doc. 1 - Le baptême de Clovis

(tiré de Pour connaître la France CM, Hachette 1985). 


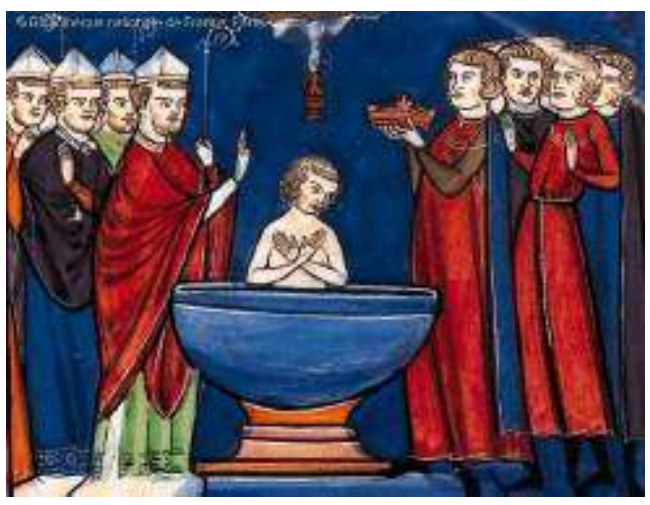

Doc. 2 - Le baptême de Clovis

(Les Grandes Chroniques de France $\mathrm{XIV}^{\mathrm{e}}-\mathrm{XV}^{\mathrm{e}}$ siècles), enluminure.

\section{NOTES}

1. Défini comme «éventuel narrateur ou personnage focal auquel on rapporte les éléments subjectifs du texte ». (p. 38)

2. Dans la transcription, $M$ renvoie à maitre, $E$ à élève. Quand l'élève est identifiable par son prénom grâce aux propos de l'enseignant, c'est l'initiale du prénom de l'élève qui est reprise.

3. Défini comme "une stratégie, pas nécessairement consciente, permettant au locuteur de donner l'impression qu'il se retire de l'énonciation, qu'il «objectivise» son discours en "gommant» non seulement les marques les plus manifestes de sa présence (les embrayeurs) mais également le marquage de toute source énonciative identifiable.» (Vion, 2001 : 334).

4. «L'enseignant l'initie (l'élève) à la méthode du questionnement et, comme dans la méthode scientifique, lui apprend progressivement à émettre des hypothèses, à privilégier la recherche du sens sur l'accumulation des faits et des preuves, à les justifier par des arguments, à y renoncer quand elles apparaissent fausses. [...] C'est une première forme, modeste mais réelle, d'esprit critique.» $(2002: 8)$

5. En 1996, on commémorait les 1500 ans de cette cérémonie (encore qu'aujourd'hui la date de 496 soit contestée), et ce que représente idéologiquement Clovis est rappelé dans un article du Monde de l'éducation(1996): "La présentation de Clovis et celle de Vercingétorix ont focalisé l'image d'une France coupée en deux jusqu'à la première guerre mondiale, [rappelle Yves Galupeau, Conservateur au Musée national de l'éducation de Rouen]. Traditionnellement, la conception confessionnelle de l'histoire affirme que la France commence quand elle est catholique : son point de départ, c'est Clovis, qui a donc été longtemps présenté en ouverture des manuels. La conception laïque et républicaine fonde l'enseignement de l'histoire sur la notion de peuple : ses manuels démarraient par Vercingétorix, en occultant Clovis, voire en oubliant le baptême. "

6. Lire pour comprendre l'évolution du baptême au sacre à propos de Clovis «Reims, ville du sacre » de Jacques Le Goff in Les lieux de mémoire sous la direction de Pierre Nora, t. II, La nation, Gallimard, 1986.

7. Il est fait mention de l'ampoule par l'évêque de Reims, Hincmar, en 878. En racontant le baptême de Clovis dans une vie de saint Rémi, il ajoute la légende du chrême apporté du ciel. Il juxtapose deux cérémonies : le baptême et le sacre. Une coutume essentielle de la royauté est née : les rois de France sont sacrés avec le chrême de la sainte ampoule qui est venu du ciel pour le baptême de Clovis. 


\title{
RÉSUMÉS
}

Les enseignants, à l'école élémentaire, utilisent fréquemment, dans leurs séances consacrées à l'histoire, des documents qu'ils traitent hors manuel. L'article propose l'analyse d'une de ces séances, filmée dans des conditions écologiques. Il montre comment la médiation opérée par l'enseignant entre les documents et les élèves n'est pas à même de mettre en place chez ceux-ci une compétence de lecture importante : celle de se construire une posture d'archi-énonciateur à même de hiérarchiser les informations livrées par les documents de façon à reformuler les points de vue en objet de savoir.

\begin{abstract}
A history lesson in cycle 3 (keystage 2): the teacher's enunciative posture in the mediation between documents and pupils

The article consists in the analysis of an ordinary history lesson in keystage 2: the collective exploitation of iconographic documents depicting Clovis' baptism. The aim of the lesson resides in fact more in the methodology than in the subject itself: it is about teaching pupils to take a critical distance when dealing with documents. But the fact that the teacher mistakes a superficial analysis of the documents for objectivity - because it influences the choice of the documents and the enunciative posture of lecturing in the planning of the lesson and the monitoring of verbal exchanges - means the pupils are not able to learn how to be critical of what they read. This oral lesson doesn't help them build the posture of an archi-enunciator who can sort out the information given in the documents so as to turn the points of view into objects of knowledge.
\end{abstract}

\section{AUTEUR}

\section{ANNE LECLAIRE-HALTÉ}

IUFM de Metz, Université Paul Verlaine, Celted. 